\title{
Webizing 3D contents for super multiview autostereoscopic displays with varying display profiles
}

\author{
Daeil Seo ${ }^{* \dagger}$ \\ Byounghyun $\mathrm{Yoo}^{\S+1}$ \\ Jonghyun Choi \\ †Korea Institute of Science and Technology "University of Science and Technology
}

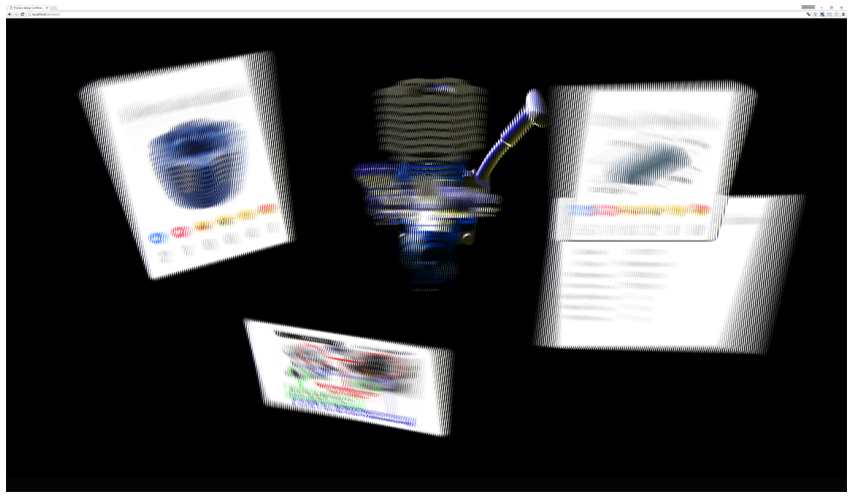

(a)

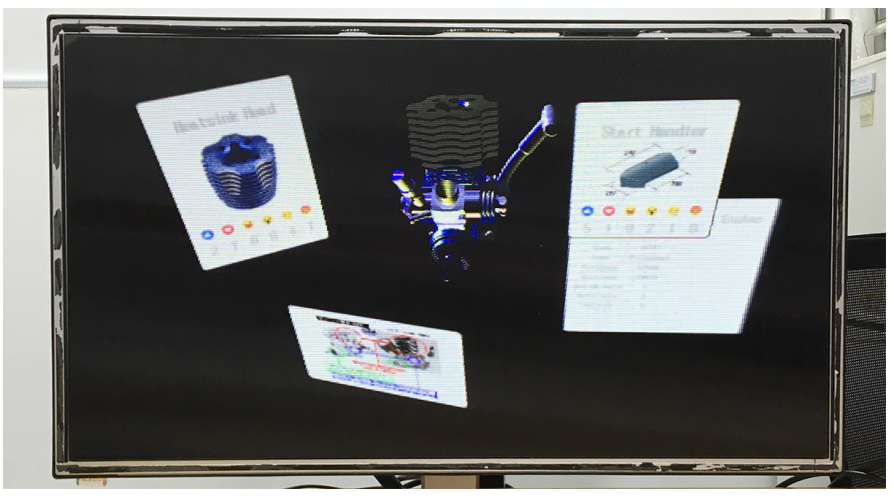

(b)

Figure 1: Webized autostereoscopic 3D content on a super multi-view display for a product design conference: (a) rendering the content on the screen and $(b)$ the user's view in front of the display using a super multi-view autostereoscopic display.

\section{Abstract}

With the development of optic technologies, various forms of stereoscopic 3D displays such, as 3DTVs, head-mounted displays, multi-view autostereoscopic displays, and super multi-view autostereoscopic displays, have emerged. However, displays have various characteristics and different implementations and the supply of content and rendering applications cannot keep up with the development speed of the displays. The content production varies and depends on the characteristics of a specific display. To reuse content with various types of autostereoscopic 3D displays with different characteristics, the content or rendering application should be modified for other displays. To resolve these problems, we propose a webizing method for the content and display of super multi-view autostereoscopic systems in the Web environment. The proposed method renders not only 3D autostereoscopic model by WebGL but also existing web pages described by HTML on the Web as super multi-view autostereoscopic 3D system content on the Web. In addition, we propose a JavaScript interface for describing displays to allow developers to access the profile and functionalities of displays through web browsers. This interface helps rendering applications generate monoscopic $3 \mathrm{D}$ or $\mathrm{N}$ multi-view autostereoscopic $3 \mathrm{D}$ content based on a display profile from the same source, also known as one-source multi-use. We present an example of super multi-view autostereoscopic content and display it in a product design conference on the Web using a prototype implementation

*e-mail: xdesktop@kist.re.kr

${ }^{\S}$ Corresponding author. e-mail: yoo@byoo.net

Permission to make digital or hard copies of part or all of this work for personal or classroom use is granted without fee provided that copies are not made or distributed for profit or commercial advantage and that copies bear this notice and the full citation on the first page. Copyrights for thirdparty components of this work must be honored. For all other uses, contact the Owner/Author.

Copyright is held by the owner/author(s).

Web3D '16, July 22-24, 2016, Anaheim, CA, USA

ACM 978-1-4503-4428-9/16/07.

DOI: http://dx.doi.org/10.1145/2945292.2945295 to verify the usefulness of our approach.

Keywords: webizing, super multi-view, content multiplexing, mixed HTML5 and WebGL, 3D Web

Concepts: • Human-centered computing $\rightarrow$ Interaction paradigms; Web-based interaction; • Computing methodologies $\rightarrow$ Computer graphics; Rendering;

\section{Introduction}

As mobile broadband technology continues to develop, the demand for high-fidelity visual content has increased. 3DTVs using a binocular parallax have not been widely accepted in the market because of the discomfort of wearing glasses and the lack of content. In contrast, glass-free super multi-view (SMV) autostereoscopic 3D displays greatly improve visual fidelity by providing motion parallax as well as improve the user's convenience by eliminating the necessity of wearing glasses. We need a method to supply content through these display devices. To date, content production and rendering applications for these displays have depended on the characteristics of a specific display. Previous works related to SMV autostereoscopic 3D displays have focused on improving the degree of hardware completion rather than content production. For this reason, by neglecting the user interface and user experience of autostereoscopic 3D content, a content ecosystem for SMV displays has not been attempted. To avoid the discontinuation of the 3DTV market, it is necessary to provide an efficient content production pipeline for autostereoscopic content and an implementation method for the user's interaction with content.

In this paper, we focus on the content production pipeline and interaction methods for SMV display applications. The method uses existing resources, such as HTML documents and 3D model files on the Web to create SMV autostereoscopic 3D content. A prototype application is implemented based on this method in a 3D Web environment. The following problems are discussed in this paper: 
- The supply of autostereoscopic 3D content: The content production depends on the characteristics of a specific display for demonstration. A general content supply method that is independent of SMV content and display is needed.

- Webizing SMV content: Currently, the Web mostly includes 2D content, such as text, pictures, and monoscopic videos. With the advent of HMD and SMV displays, 2D content must be elevated to a minimum of $2.5 \mathrm{D}$ content for the stereo of HMD displays and perhaps even full 3D content for SMV displays. Thus, webizing SMV content may involve intermixing 2D content as well 3D content seamlessly.

- Webizing SMV display: Previous rendering applications cannot access the characteristics of SMV displays. If the applications can access display characteristics, they can generate tailored rendering content dynamically from the same content source. The declaration of an SMV display characteristics profile and interface for accessing the profile of SMV displays on the Web is necessary.

To resolve these problems, HTML document object model (DOM) elements and 3D models share a 3D layout space and are simultaneously rendered in a single scene. The scene should be rendered on the screen based on the characteristics of the given SMV autostereoscopic displays. In this paper, we propose a device interface model for SMV autostereoscopic displays to render tailored SMV content to the display. Additionally, we propose a webizing method for SMV content that contains HTML DOM elements and 3D models on a shared 3D layout on the 3D Web. A prototype application based on our proposed method renders a monoscopic 3D content, stereoscopic 3D content, and SMV stereoscopic 3D content from the same source content according to the characteristics of the given display using standard web technologies without additional browser plug-ins. The SMV content is described in a HTML document by the declarative method [W3C Community Group 2013]. To handle events for 3D assets (i.e., 3D models and HTML DOM elements in a HTML document) on 3D content, developers describe event handlers according to W3C's DOM events specification.

The content developers of an SMV autostereoscopic 3D system can reuse existing content and libraries on the web for creating SMV 3D content. This can resolve the issue of a lack of SMV 3D content. The applications create SMV 3D content dynamically on the web browsers using original content based on the device interface model from the SMV display. The remainder of this paper is structured as follows. We present supporting related works on content and displays in Section 2, after which we introduce the proposed webizing super multi-view autostereoscopic method on the 3D Web in Section 3. Then, we explain the prototype implementation in Section 4. We present the experimental results to verify the proposed method and discuss lessons learned in Section 5. Finally, we conclude with a summary and an outlook for future work in Section 6.

\section{Related Work}

\subsection{Super multi-view autostereoscopic display}

Urey et al. [2011] examined most of the 3-D displays and presented recent developments and advances. In principle, an autostereoscopic direct-view display can take many forms, ranging from two-image stereo, multi-view, and super multi-view (SMV). These systems use technique for creating the illusion of three-dimensional depth by fusing together images in the human brain from two-dimensional images viewed by the eyes. Twoview display systems produce a single pair of parallax views for binocular vision at a single location. The multi-view displays present a series of a relatively small number of views with fixed viewing zones. The number of views of the displays is too small for continuous motion parallax. The simple approach creates twoimage stereoscopic content at each of viewpoints (i.e., the positions of the left and right eyes) and renders the content on offscreen. The approach cuts a source image into multiple strips according to the number of viewpoints depending on a display and merges these strips sequentially into a single displayed image, as shown in Figure 2. The stereoscopic content using this spatially multiplexed image-interleaving algorithm has half the resolution of the original images.

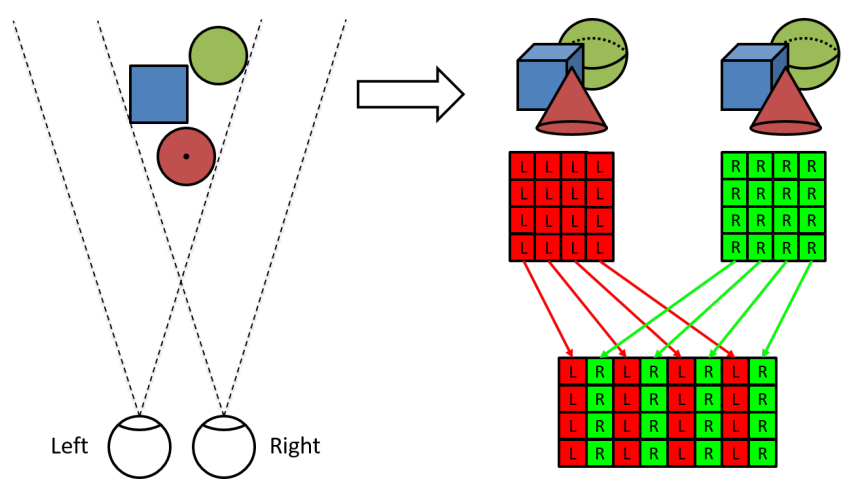

Figure 2: Overview of creating two-image stereoscopic content

The SMV autostereoscopic display shows stereoscopic content by spatially-multiplexed image interleaving without the use of any eyewear. The SMV displays are implemented using parallax barriers or the lenticular screens method. The parallax barriers are located in front of an image source and allow light to pass only to the desired viewing zone through black masks. However, this approach has a loss of brightness problem. On the other hand, the lenticular screens with cylindrical lenses can be used to direct the diffused light from a pixel. It can only be seen at a certain viewing angle in front of the display. The slanted lenticular system allows for the interspersing of odd and even views, and the system has minimal resolution loss by dispersing resolution loss in two directions [Berkel 1999].

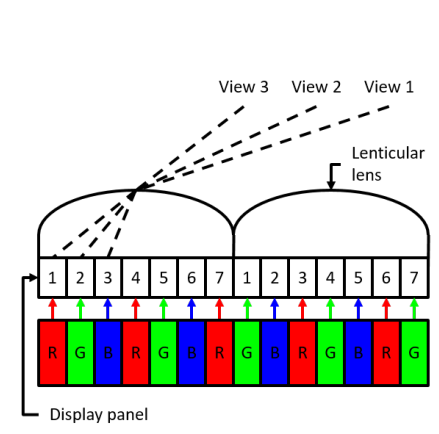

(a)

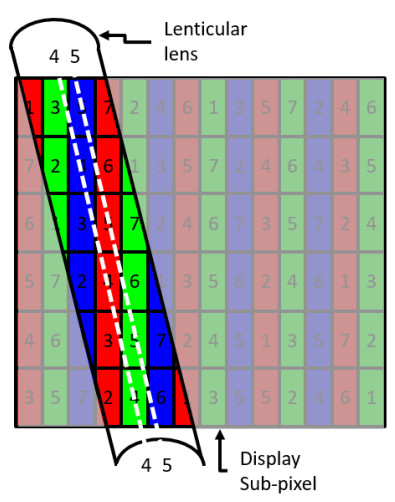

(b)
Figure 3: Pixel arrangement of 7-view slanted lenticular display: (a) upper view and (b) front view.

Figure 3 is an example of the slanted lenticular multi-view display using a spatial multiplex design. Each number denotes an image 
corresponding to a specific viewpoint. The lenticular lenses are aligned with the vertical pixel columns in front of a 2D display screen, as shown in Figure 3(a). Each pixel column is assigned to a single view, and the user achieves one pixel by merging subpixel scale's R, G, and B channels on the slanted lenticular lens. Figure 3(b) depicts the relationship between the sub-pixels and the slanted lenticular sheet. The horizontal position of the sub-pixels depends on the viewing angle. In this paper, we deal with a slanted lenticular SMV display and the rendering method for the display will be described in Section 3.

\section{$2.23 \mathrm{D}$ content rendering on the Web}

Two kinds of approaches have been used to render a 3D model on the 3D web. Using procedural APIs, Web Graphics Library (WebGL) provides an API written in JavaScript for rendering 3D graphics within any compatible web browser without the use of plug-ins [Parisi 2012]. Three.js is a JavaScript 3D library using WebGL to create and display 3D computer graphics in a web browser [Dirksen 2013]. Another approach is the declarative 3D method. X3DOM [Behr et al. 2009] or XML3D [Sons et al. 2010] creates 3D objects by adding document object model (DOM) elements as part of the HTML document. Declarative 3D for the web architecture of the $\mathrm{W} 3 \mathrm{C}$ community group is used to determine the requirements, options, and use cases for $3 \mathrm{D}$ graphics capabilities in a declarative way [W3C Community Group 2013]. To render a 3D scene using DOM elements in HTML documents, Fraser et al. [2013] proposed cascading style sheets (CSS) transforms that allow the transformation of HTML DOM objects in two-dimensional or three-dimensional space. Ahn et al. [2014] extended CSS page media to CSS place media to support the rendering of an HTML document as a 3D volumetric medium. Seo et al. proposed a system to render HTML DOM elements and 3D objects in a shared 3D layout for 3D web [Seo et al. 2015] and mixed and augmented reality [Seo et al. 2016] environments.

To render two-view stereoscopic 3D content on the Web, the applications render 3D models from files for the declarative method or JavaScript code for the procedural method as shown in Figure 4(a). The application renders a scene on off-screen with the camera located at the first viewpoint and moves the camera position to offset of viewpoints. The application generates an image on each $\mathrm{N}$ viewpoint. A fragment shader multiplexes images into a final scene according to the characteristics (e.g., sub-pixel arrangement, number of viewpoints, and screen resolution) of the slanted lenticular display and renders the final scene. Johnston et al. [2013] developed interactive stereoscopic 3D content for biomedical visualization using WebGL. Three.js provides various effects such as stereoscopic, cardboard, and parallax barrier effects for creating stereoscopic 3D content from monoscopic 3D content [Dirksen 2013]. An alternative approach is to create stereoscopic 3D content from HTML DOM elements in web pages by extending existing web technologies, as shown in Figure 4(b). These methods use CSS 3D Transforms [Fraser, et al. 2013] for the arrangement of HTML DOM elements on 3D space and generate a duplicate of the original content. Finally, the twoview content is rearranged according to the eye offset for a stereoscopic effect. Chistyakov et al. [2013] presented 3DSjQ, a 2D-to-3D conversion algorithm for achieving stereoscopic 3D in web pages using HTML5, CSS3, and jQuery. Chen et al. [2014] implemented stereoscopic 3D using the WebKit browser by adding new attributes to HTML, CSS, and JavaScript. Lee et al. [2015] extended CSS specifications to support stereoscopic 3D web content written in HTML5 and showed examples of content on a stereo device. González-Zúñiga and Carrabina [2015] developed a toolkit to create stereoscopic side-by-side 3D content using the HTML5 canvas element. However, these works render two-view stereoscopic 3D content and use either a 3D model or HTML DOM elements.

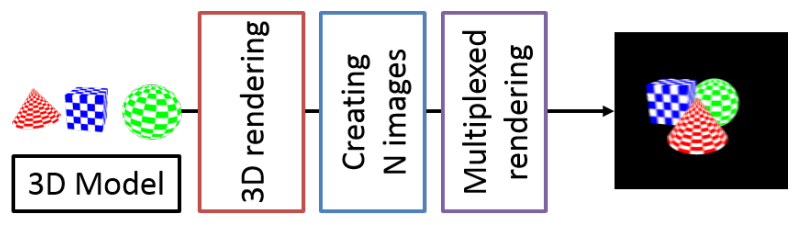

(a)

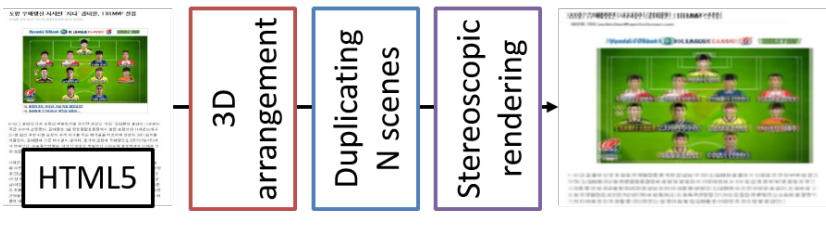

(b)

Figure 4: Previous methods for multi-view autostereoscopic content: (a) stereoscopic $3 D$ content using WebGL and (b) stereoscopic 3D HTML

To render $\mathrm{N}$ multi-view stereoscopic content, the applications render $\mathrm{N}$ images on off-screen by moving the camera position of the $\mathrm{N}$ viewpoints and multiplex the images to a content on screen. Kooima et al. [2007] proposed an autostereoscopic rendering algorithm using a graphics processing unit (GPU) for vertex and fragment processing with OpenGL Shading Language (GLSL). Sorbier et al. [2008] used geometry shaders to take advantage of the rendering process by duplicating and transforming primitives to the desired viewpoints. Ahn and Kim [2009] implemented a GPU-based rendering system for a multiple-view autostereoscopic display to present seamless 3D stereo experiences from an arbitrary observer's viewing position. However, these works do not work on the Web because they are implemented using OpenGL in native environments. In addition, these methods cannot generate SMV autostereoscopic content using HTML DOM elements because the GLSL code in the vertex and fragment shaders properties must be compiled and run on the GPU using WebGL. Kim et al. [2012] simulated image distortion results according to variations in an observer's viewing orientation and subsequent compensation in a stereoscopic display.

\subsection{D display interface on the Web}

To render SMV content on the Web, the content should be described by web technologies and the rendering applications also need a method to access the display profile and functionalities on the Web. W3C's device APIs and policy working group published the requirements of device APIs for enabling web applications to interact with device functionalities [Berjon et al. 2009]. Regarding 3D display devices' view, Han and Lee [2012] proposed extensions for the stereo 3D web to render stereo views of 3D objects on web browsers with 3D displays. They extended CSS 3D Transforms, added the "stereo" attribute in the HTML tag for image and video objects, and proposed the "3d-display" media type for media queries. They also introduced "Display Interface" which is a Device API for stereo rendering to check the stereo3D-capable display and the 3D mode. The stereoscopic 3D Web community group of $\mathrm{W} 3 \mathrm{C}$ is focused on determining the requirements, available options, and use cases for the stereoscopic 3D Web [W3C Community Group 2013]. Vukicevic et al. [2016] 
proposed a WebVR specification for describing VR hardware to allow developers to access VR devices such as the Oculus Rift or Google Cardboard, in their web browsers. They define DOM Interfaces (e.g., VRDisplay, VRDisplayCapabilities, and VRFieldOfView) to support runtime access to the functionalities of devices. W3C Community Group [2016] also started the WebVR community group activity to help bring highperformance VR to the open Web. However, these works consider supporting only stereoscopic display devices on the Web. To render SMV autostereoscopic content on the 3D Web, an interface for SMV displays in a similar way is requried.

\section{$3 \quad$ Webizing SMV autostereoscopic system}

Berners-Lee [1998] mentioned that webizing is a way of bootstrapping the web using a large amount of legacy information, and the essential process in webizing is to take a system that is designed as a closed world and then ask what happens when it is considered as part of an open world. Our proposed webizing method for SMV autostereoscopic system consists of webizing for displays and contents. The webizing display abstracts various SMV display devices by providing a JavaScript interface to access display profiles. The webizing SMV content uses unified content description for 3D assets on shared 3D layout and enables content presentation from a single source in multiple presentations according to display profiles.

\subsection{Webizing display}

The SMV autostereoscopic display shows the 3D stereoscopic content that is generated according to the characteristics, such as the number of viewpoints and the offset between the viewpoints of the display. The application creates $\mathrm{N}$ images and performs a spatially multiplexed image-interleaving algorithm to render final content from $\mathrm{N}$ images. The number $\mathrm{N}$ is the number of viewpoints in the display profile. To render 3D stereoscopic content on the 3D Web, the application can access the display specification by JavaScript because the forms of SMV displays are various and different. W3C proposed the requirements of device APIs to access device functionalities for developers [Berjon, et al. 2009]. The WebVR interface is a method to access the specifications of VR devices [Vukicevic, et al. 2016].

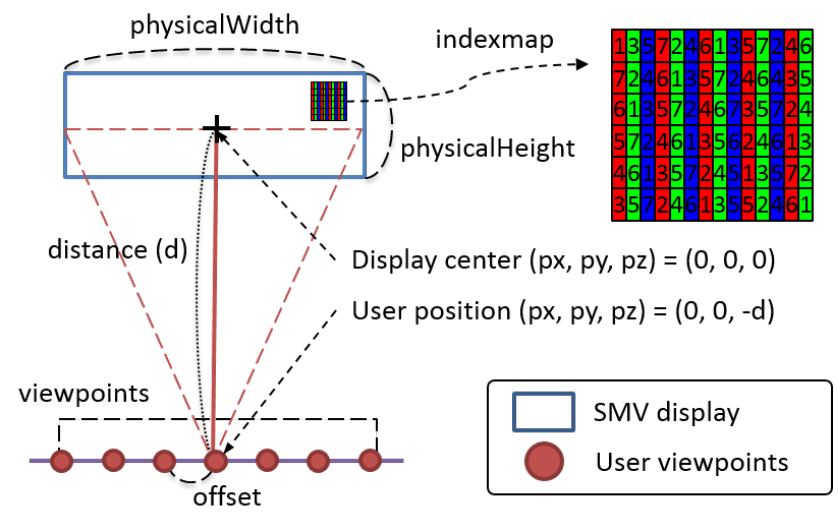

Figure 5: Overview of SMV autostereoscopic display

Figure 5 depicts an example environment of an SMV autostereoscopic display. The SMV autostereoscopic display shows the 3D stereoscopic content on the screen and the user is located at a distance. The distance depends on the optical crosstalk of the SMV display. The display also has $\mathrm{N}$ viewpoints and the offset according to the arrangement of lenticular lenses. The characteristics of SMV autostereoscopic displays affect the rendering of $3 \mathrm{D}$ stereoscopic content. In this paper, we define a JavaScript interface for a webized super multi-view display (SMVDisplay), as shown in Table 1. The profile of the SMV display includes the physical size and resolution of the screen to determine the sizes and positions of $3 \mathrm{D}$ assets on the shared 3D layout. The viewpoints attribute is the number of viewpoints, and the offset attribute is the distance between viewpoints for the camera position on $\mathrm{N}$ viewpoints. The distance attribute is a place where the user has the minimal optical crosstalk of the SMV display. The indexmap attribute is used for spatially multiplexed image-interleaving to denote the index of the images and position of the sub-pixels in the selected image. The indexmap is a twodimensional array and the size of the array is determined by [screenWidth * 3] [screenHeight] because the SMV display uses separated R, G, and B channels in pixels according to the arrangement of the slanted lenticular lenses.

Table 1. Webized SMVDisplay interface

\begin{tabular}{|l|c|l|}
\hline \multicolumn{1}{|c|}{ Attribute } & Type & \multicolumn{1}{c|}{ Description } \\
\hline physicalWidth & float & $\begin{array}{l}\text { Physical width size (mm) of the screen of } \\
\text { a display }\end{array}$ \\
\hline physicalHeight & float & $\begin{array}{l}\text { Physical height size (mm) of the screen of } \\
\text { a display }\end{array}$ \\
\hline screenWidth & integer & Number of width pixels (px) in the screen \\
\hline screenHeight & integer & $\begin{array}{l}\text { Number of height pixels (px) in the } \\
\text { screen }\end{array}$ \\
\hline viewpoints & integer & Number of viewpoints \\
\hline offset & float & $\begin{array}{l}\text { Distance (mm) of neighborhood } \\
\text { viewpoints }\end{array}$ \\
\hline distance & float & Distance (mm) between user and display \\
\hline indexmap & $\begin{array}{c}\text { integer } \\
\text { [][] }\end{array}$ & $\begin{array}{l}\text { Subpixel index for spatially multiplexed } \\
\text { image-interleaving multi-view content }\end{array}$ \\
\hline
\end{tabular}

\subsection{Webizing content}

The SMV autostereoscopic 3D content is generated by SMV applications using monoscopic 3D content. To use 3D models and HTML DOM elements for monoscopic 3D content, our proposed method uses the webizing 3D method [Seo, et al. 2015] for sharing a 3D layout between 3D models and HTML DOM elements on the $3 \mathrm{D}$ web. The webizing $3 \mathrm{D}$ method only deals with monoscopic 3D content on the 3D web. In this paper, we propose a webizing SMV 3D method that extends the webizing $3 \mathrm{D}$ method to the content and display of the SMV system. The proposed method describes HTML DOM elements and 3D models in a HTML document and uses CSS3 for the arrangement of shared the 3D layout.

To declare 3D models in the HTML document, the proposed method needs a way of linking external 3D model files. We use a href attribute of an anchor tag $\langle a\rangle$ to denote a URL of the 3D model file. The type attribute specifies the media type of the 3D model file. The proposed method can support all 3D model types if the application has the decoders of the 3D model types. We also use "--smv-transform" CSS extensions for the arrangement of 3D assets in the shared 3D layout. The notation of our proposed CSS extensions is based on CSS 3D Transforms [Fraser, et al. 2013]. The proposed method applies translate, rotation, and scale values using a CSS selector to $3 \mathrm{D}$ assets when they are rendered on the screen. The differences between CSS 3D Transforms and our proposed method are given in Table 2 . 
Table 2. Comparison of CSS 3D Transforms and our method

\begin{tabular}{|l|l|l|}
\hline $\begin{array}{c}\text { 3D Transform } \\
\text { Methods }\end{array}$ & \multicolumn{1}{|c|}{$\begin{array}{c}\text { CSS 3D Transforms } \\
\text { (transform property) }\end{array}$} & $\begin{array}{c}\text { Our proposed method } \\
\text { (--smv-transform property) }\end{array}$ \\
\hline translate & pixel value & numerical value \\
\hline scale & numerical value & numerical value \\
\hline rotate & degree & radian \\
\hline
\end{tabular}

Figure 6 depicts an example HTML document of webized SMV content using the declarative method. The 3D transform information is defined by CSS, and the property name is "--smvtransform". The value of the property has the same notation as CSS 3D Transforms. However, the unit of the value is different. In this example, two HTML DOM elements and two 3D models are declared. The anchor tags denote the URL of the 3D model files. The 3D model is loaded using the href attribute's value of the anchor tags, and the model is rendered by a mime-type that is declared in the type attribute. The result of monoscopic rendering is shown in Figure 7(a). To process events for 3D models in the HTML document, the proposed model uses W3C's DOM Level 3 [Hors et al. 2004]. The developer can write the routines of event handling with JavaScript, which is similar to the event handling for HTML DOM elements on the web browsers. In this example, when the user clicks on a 3D model for which the id attribute is "cube", a visible status of a HTML DOM element, for which the id attribute is "annotation-cube", is toggled.

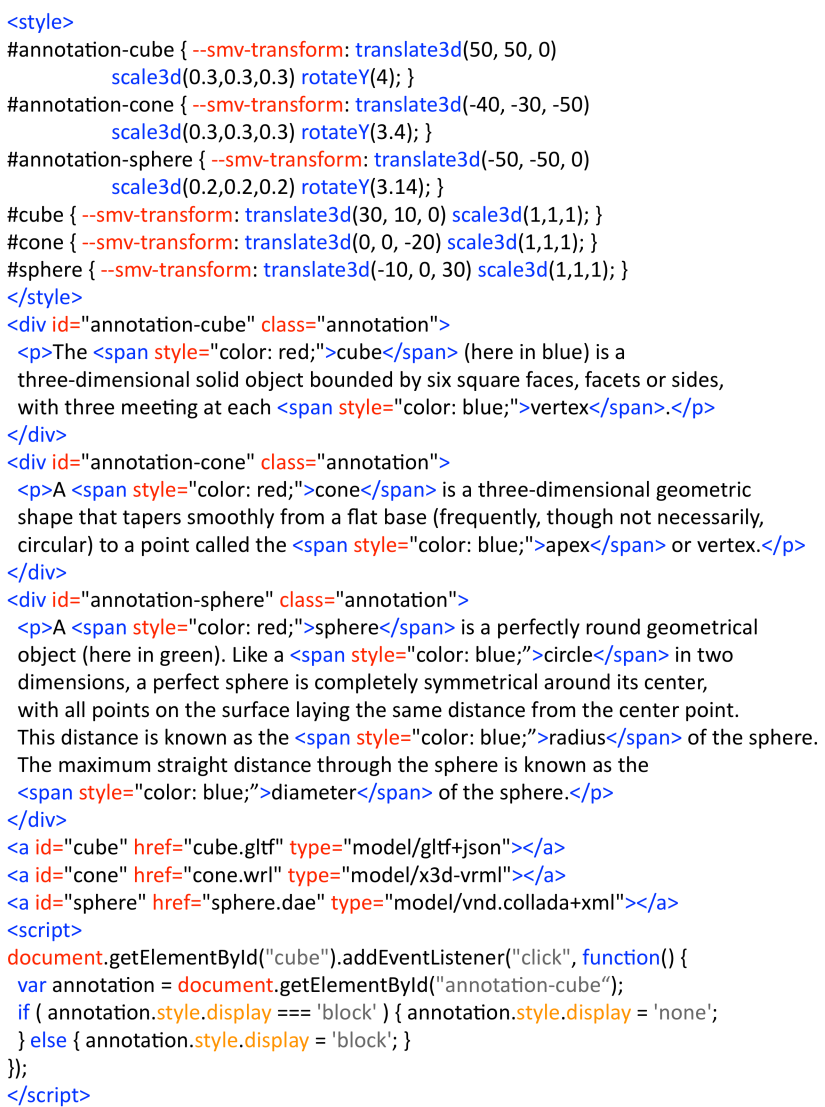

Figure 6: Example of webized SMV content

In the rendering result, the $3 \mathrm{D}$ assets are arranged in a shared 3D layout. To render the content for an SMV display, a method is needed for spatially multiplexed image-interleaving from a HTML canvas DOM element for WebGL and other HTML DOM elements. Figure 7 shows difference of 4 multi-view rendering examples of the SMV content given in Figure 6. Figure 7(a) is a monoscopic rendering result, and the other results used it to render a scene on different viewpoints. They move the camera position four times according to the eye's offset and perform the image-interleaving method to render a final scene. Figure $7(\mathrm{~b})$ is a stereoscopic rendering result based on Figure 4(a) that affects only the 3D model in the scene. On the other hand, Figure 7(c) depicts a stereoscopic 3D HTML result based on Figure 4(b) that affects only HTML DOM elements except the HTML canvas element for WebGL. To obtain SMV stereoscopic content, as shown in Figure 7(d), a method is needed for rendering 3D models and HTML DOM elements together.

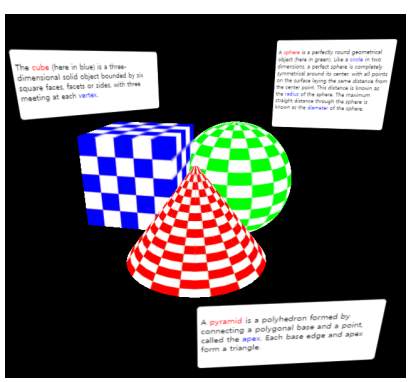

(a)

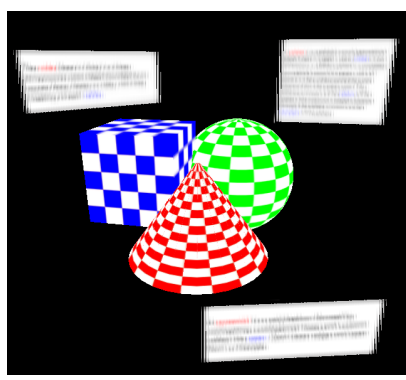

(c)

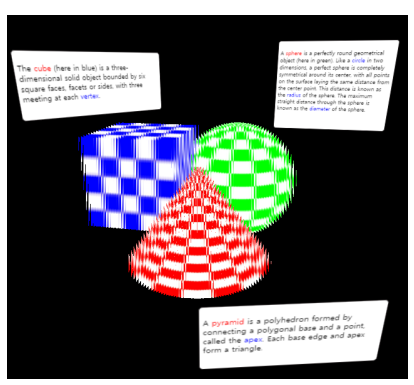

(b)

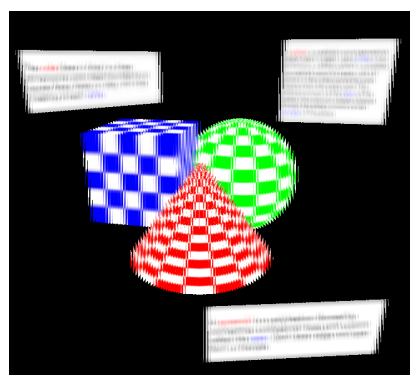

(d)
Figure 7: Results of spatially multiplexed image-interleaving for 4 multi-view $3 D$ content on the web: (a) monoscopic mixed $3 D$ assets, (b) $3 D$ stereoscopic content using WebGL, (c) $3 D$ stereoscopic HTML, and (d) SMV 3D stereoscopic content

To resolve this problem, the $3 \mathrm{D}$ assets should be rendered on a single scene in the web browsers without additional plugins or modification. Webizing SMV content involves intermixing 2D content as well 3D content seamlessly. The proposed method generates an image from the HTML DOM element and adds a plane geometry to the image in the scene for 3D models. The plane geometry has the same size, position, and transform values as the source HTML DOM element. When the source HTML DOM element is changed, the image is also updated. Through this approach, the proposed method adds a component for blending the $3 \mathrm{D}$ models and HTML DOM elements in the single 3D model scene, as shown in Figure 8 . The display profile provides a characteristic of the SMV display that helps to determine the position and size of the HTML DOM elements in the 3D model scene. This is a different point from that of Figure 4(a) and (b). The processes after the blending process are similar to those of Figure 4(b). The proposed method creates $\mathrm{N}$ images by moving the camera position to offset according to the display profile, as shown in Table 1. Finally, the method generates the content by multiplexing $\mathrm{N}$ images based on the indexmap in the display profile and renders the content on the screen. 


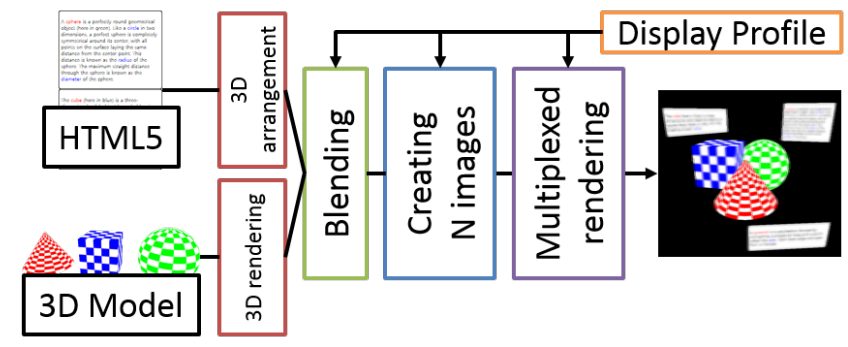

Figure 8: Webized rendering outline of SMV stereoscopic content

\section{Prototype Implementation}

We implemented a prototype system for webized SMV autostereoscopic displays. Figure 9 shows an overview of the prototype system architecture. The prototype implementation consists of four parts: the display profile, the content store provider, the 3D content renderer, and the SMV content renderer. The display profile is a JavaScript interface for accessing a display profile and functionalities on the web browsers. The content store provider manages HTML documents that contain HTML DOM elements and links for 3D model files. The 3D content renderer requests a HTML document and 3D model files, and generates a 3D integrated scene graph for the SMV content renderer. The webized $3 \mathrm{D}$ content loader running on the web browsers requests the HTML document to the content store provider by the HTTP GET method. The content store provider sends the requested HTML document, and the webized 3D content loader finds links for 3D model files using anchor tags. The loader additionally requests the $3 \mathrm{D}$ model files from the content provider. The loader parses the HTML document and 3D model files and generates the webized 3D scene graph. The scene graph has HTML DOM elements from the HTML document and $3 \mathrm{D}$ models from the $3 \mathrm{D}$ model files. The $3 \mathrm{D}$ scene graph uses the display profile to rearrange the HTML DOM elements on the shared 3D layout. The webized 3D blender integrates the 3D assets into a single scene graph. The result is delivered to the SMV content renderer.

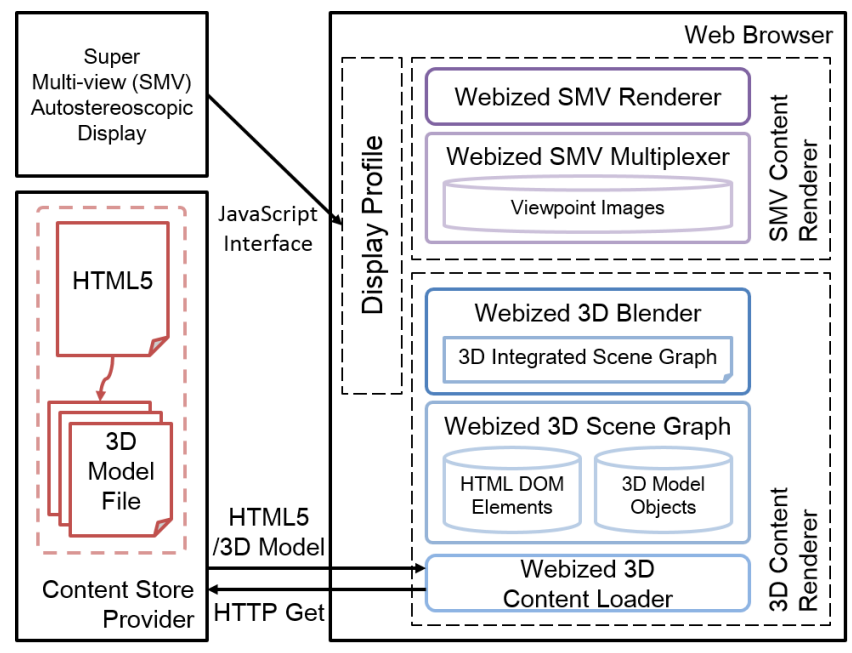

Figure 9: Overview of prototype system architecture for rendering webized SMV autostereoscopic content

The SMV content renderer creates $\mathrm{N}$ images by moving the camera position to the $\mathrm{N}$ viewpoints using the $3 \mathrm{D}$ integrated scene graph. The SMV content renderer performs the spatially multiplexed image-interleaving algorithm to generate the final content for the display. The detailed process proceeds as follows. The webized multiplexer obtains the display profile, as shown in Table 1, from the SMV display. It moves the camera position $\mathrm{N}$ times using the offset of viewpoints for creating $\mathrm{N}$ viewpoint images. The multiplexer generates the final content based on the indexmap of the display profile. The webized SMV renderer shows the content on the screen.

The event handling for HTML DOM elements on 3D Web is used in the same way as that on the Web. The elements do not need an additional method or modification for event handling because they still have their properties. In the case of 3D models, when the user invokes an event, such as by clicking or double-clicking on the model, the prototype implementation delivers the event based on W3C's DOM Level 3 [Hors, et al. 2004] to the declared element, which is linked by an anchor tag, as shown in Figure 6. The prototype handles the event using JavaScript.

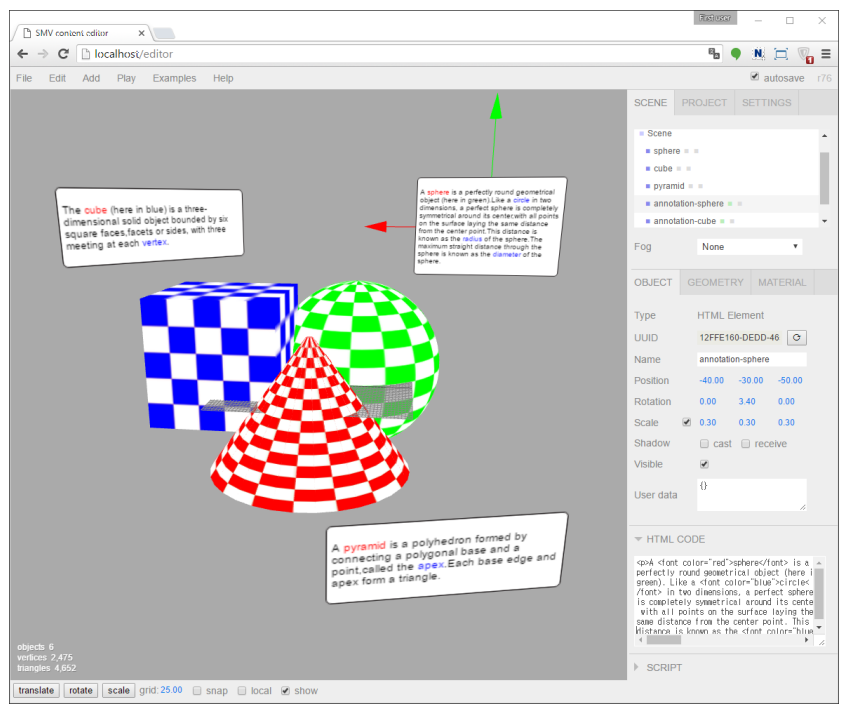

Figure 10: Webized SMV content editor

The implementation also provides an SMV content editor to create SMV content using 3D models and HTML DOM elements. It adds extensions for SMV display based on the three.js editor [Cabello 2012] which only supports 3D models, while a user cannot deal with HTML DOM elements in a 3D Web environment. The SMV editor supports 3D models and HTML DOM elements on a shared 3D Web environment. It has the 3D content renderer component, as shown in Figure 9, to support blending, as shown in Figure 8. The SMV editor enables importing and exporting 3D assets as an HTML document that describes SMV content similar to as in Figure 6. The developer can manipulate SMV content in a monoscopic 3D environment. Figure 10 depicts the SMV editor editing the SMV content shown in Figure 6 and Figure 7(a). The developer publishes the SMV content by exporting function, and a user sees the SMV content on the web browsers in the SMV display.

\section{Experimental Results and Discussion}

\subsection{Experimental Results}

We applied the prototype system to various usages of SMV content on 3D Web using SMV displays to verify the usefulness of our approach. Table 3 shows the properties of the displays used in the experiment. The experiment was performed using Intel's i72600k CPU and NVIDIA's GTX 980 GPU on Google Chrome 
web browser version 49.0.2623.112 (64-bit). Figure 1 depicts a product design conference example using the prototype implementation as shown in Figure 9. The example has a 3D model for rendering a virtual product in the center of the scene. HTML DOM documents are surrounding the 3D model and they are used for adding opinions or related content that includes multimedia content or existing web documents. The SMV content is published by the content editor as shown in Figure 10. Figure 1(a) shows a rendering result of a SMV a 3D content on the screen without lenticular lens. The display has the same physical size and screen resolution as the SMVD-Pro as given in Table 3.

Table 3. The properties example of SMV displays

\begin{tabular}{|l|c|c|}
\hline \multirow{2}{*}{\multicolumn{1}{|c|}{ Attribute }} & \multicolumn{2}{|c|}{ SMV display name } \\
\cline { 2 - 3 } & SMVD-40 & SMVD-Pro \\
\hline physicalWidth $(\mathrm{mm})$ & 640 & 621 \\
\hline physicalHeight $(\mathrm{mm})$ & 405 & 341 \\
\hline screenWidth $(\mathrm{px})$ & 2560 & 3840 \\
\hline screenHeight $(\mathrm{px})$ & 1600 & 2160 \\
\hline viewpoints & 40 & more than $60^{*}$ \\
\hline
\end{tabular}

The exact number of viewpoints for SMVD-Pro is classified by a nondisclosure agreement.

The prototype implementation draws sub-pixels separately based on indexmap in the display profile. A user can see the final result as shown in Figure 1(b), when the user is standing one meter from the SMVD-Pro display. When participants click the emoticons on the HTML DOM elements to express their opinions, the number of opinion is reflected in real time on the rendering result. If the user moves in given viewpoints of the display, the user can see seamless SMV content using motion parallax. In addition, the user interacts with the content to change rotation, position, or scale of the $3 \mathrm{D}$ assets in the scene during the product design conference. The user also changes visible status of the 3D assets and adds opinions regarding the 3D model using HTML language.

Another example shows different rendering result from the same source content because of the difference of the display profiles. Figure 11 is an example showing the solar system and planets to educate students. The crucial difference between two displays, as given in Table 3, is the number of viewpoints. Figure 11(a) is a result on the screen (without lenticular lenses), and Figure 11(c) is a result on the display using SMVD-40. Figure 11(b) and (d) show another result using SMVD-Pro. The prototype system loads a display profile by JavaScript interface when the system is initialized. The final content is different because the system generates the content dynamically according to the display profile. By these examples, we show that webized content and display is suitable for various display environments on the $3 \mathrm{D}$ web with the current standard web technologies. Figure 12 shows an example

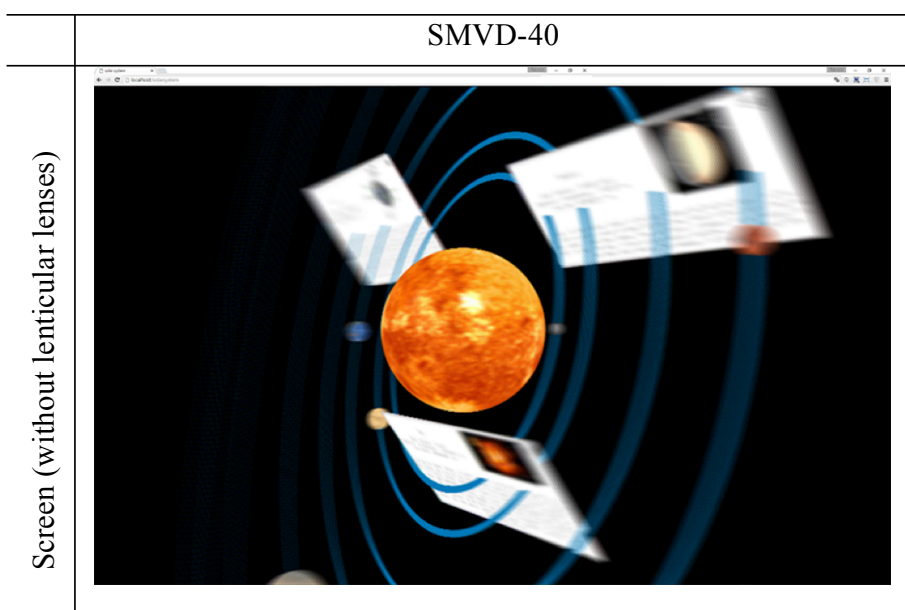

(a)

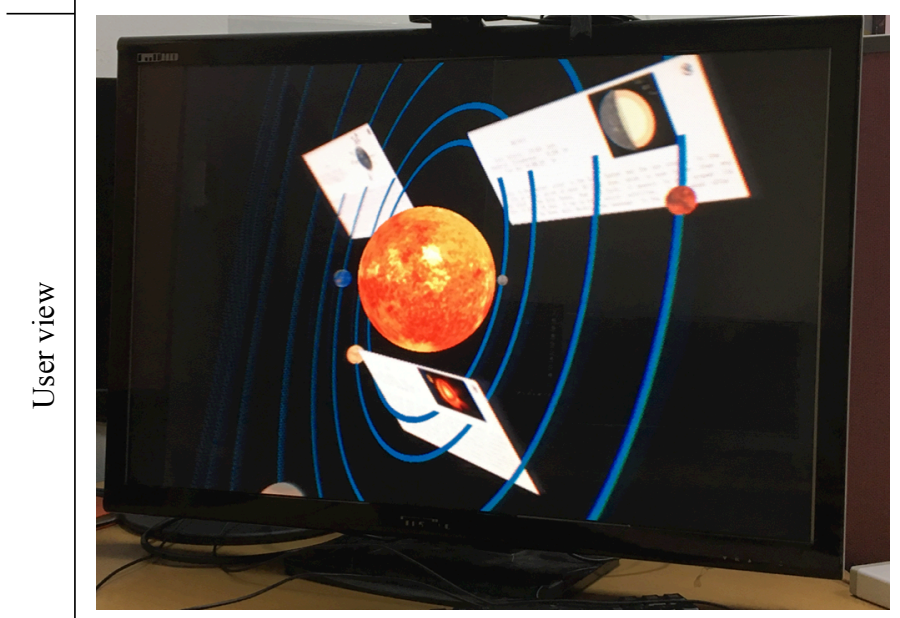

(c)

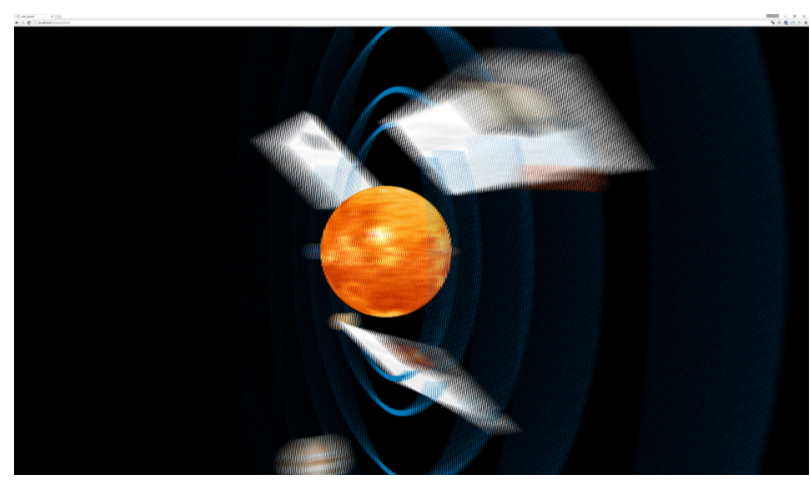

(b)

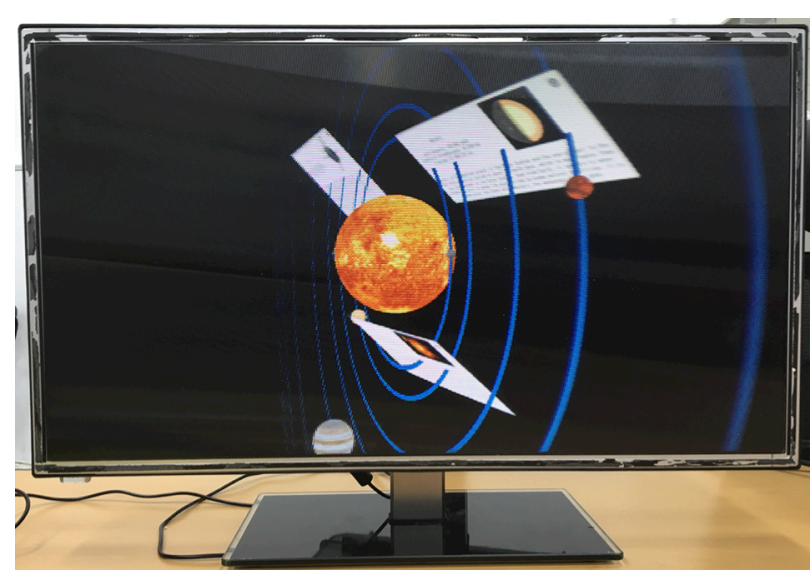

(d)

Figure 11: Rendering of the same webized SMV content on different environments: (a) SMV content with 40 viewpoints on the screen (without lenticular lenses), (b) SMV content with more than 60 viewpoints on the screen, (c) SMV display with 40 viewpoints, and (d) $S M V$ display with more than 60 viewpoints. 


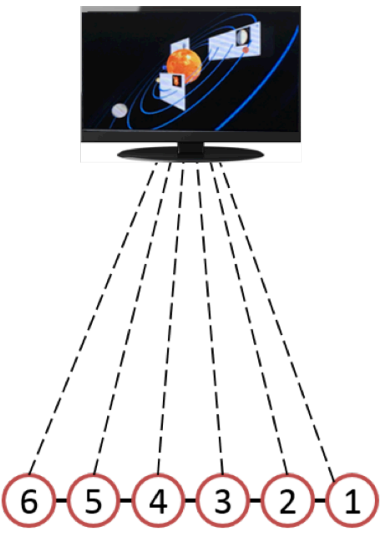

(a)
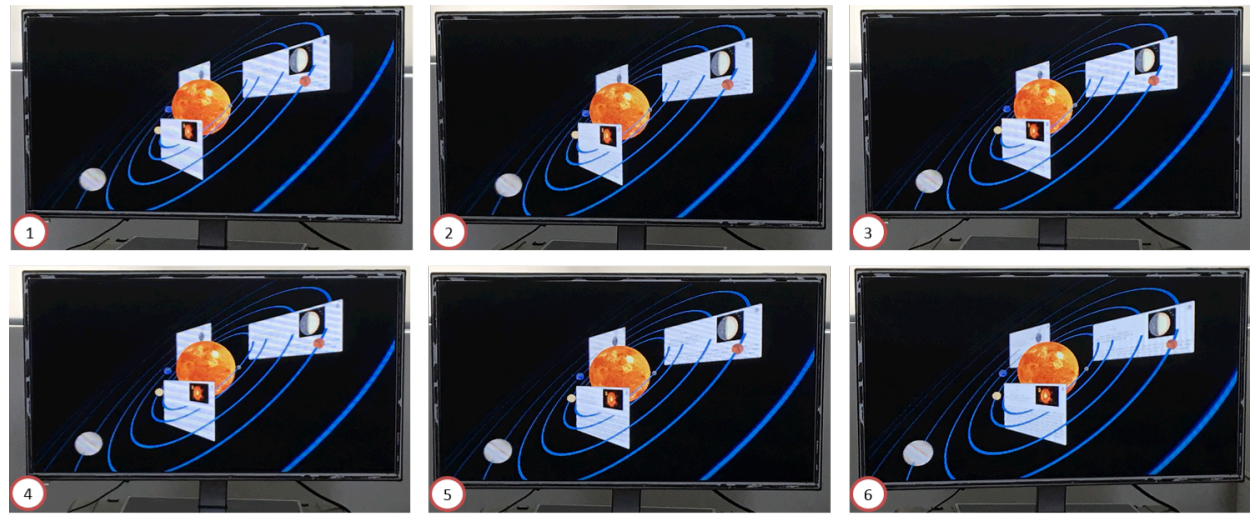

(b)

Figure 12: Motion parallax example of using SMV display with more than 60 viewpoints: (a) the movement trajectory of the user and

(b) user's views at each position.

of motion parallax result according to the user's movement. The user can view the seamless SMV 3D content in the viewing zone using motion parallax. As the user moves, the rendering result looks different and the user feels motion parallax because of the lenticular lens on the display. In this example, the Sun is located in the center of the scene. When the user moves from left to right, the distance gap between the Sun and other 3D assets (i.e., other planets and annotations) becomes narrowed. When the user moves from right to left, the distance gap between the sun and other 3D assets becomes wider inversely.

\subsection{Discussion}

To verify the usefulness of our proposed system, we discuss the advantage of the webized SMV autostereoscopic system on 3D Web compared to those of the previous approaches mentioned in Section 2 .

Rendering SMV autostereoscopic contents on 3D Web: previous works using web technologies, as discussed in Section 2.2, are focused on creating two-view stereoscopic 3D contents. They only deal with either 3D models or HTML documents for stereoscopic 3D contents. For instance, Lee, et al. [2015] proposed CSS extensions for stereoscopic 3D HTML content, as shown in Figure 4(b), and modified a standard web browser to support their proposed method. Our method renders SMV 3D content from 3D contents in shared depth buffer on 3D Web without additional plug-ins or modification of the web browsers. The $3 \mathrm{D}$ contents are interpreted on run-time and the prototype implementation renders contents according to the display profiles. The proposed method can render SMV contents in a variety of SMV display environments without additional costs compared to previous studies.

Reusability of SMV autostereoscopic contents on 3D Web: Previous works are implemented using OpenGL, which includes code for handling user interactions. It is hard to reuse 3D contents and change interaction handlers for $3 \mathrm{D}$ assets. The proposed method provides easy ways to reuse $3 \mathrm{D}$ contents or interaction handlers. The method separates the 3D contents into HTML for 3D assets, JavaScript for user interactions, and CSS for 3D transform. This point has the advantage of enabling easy reuse and change of the contents. Web developers can easily create SMV 3D contents using existing web resources because the method is based on current standard web technologies. Thus, the proposed method leverages existing web technologies and media resources to create SMV contents for SMV displays on the 3D Web.

\section{Conclusion}

In this study, we propose a webizing method for $3 \mathrm{D}$ content and display of the SMV autostereoscopic system on 3D Web. The proposed method is generalized to cover a variety of 3D displays. We present typical cases of SMV 3D contents on the 3D Web using a prototype implementation to verify the usefulness of our approach. The proposed webizing method provides a way to access the profiles of SMV displays for dynamic content rendering based on the profiles. The method reuses web documents that already exist on the web to generate SMV 3D contents. The method can use user interactions defined by JavaScript in the existing web documents without any additional event handlers. The event handling process of $3 \mathrm{D}$ models in the SMV content is similar to the event handling of HTML DOM elements. Thus, it is very convenient for web developers to easily implement user interactions for 3D content. There are no limitations to use existing sophisticated media resources and libraries on the Web. The proposed method accepts the SMV 3D system in the web environment, and the SMV 3D content production, supply, and consumption can leverage the Web. We expect that SMV 3D content will prove effective in dramatic expansion by user's participation, as with Web 2.0.

\section{Acknowledgements}

This research is supported in part by the Korea Institute of Science and Technology (KIST) Institutional Program (Project No. 2E26020).

\section{References}

AHN, J.-G. AND KIM, J. 2009. A GPU based Rendering Method for Multiple-view Autostereoscopic Display. Journal of the HCI Society of Korea 4, 9-16.

Ahn, S., Ko, H. AND YoO, B. 2014. Webizing mobile augmented reality content. New Review of Hypermedia and Multimedia 20, 79-100.

BeHr, J., EsChler, P., Jung, Y. AND ZÖLlner, M. 2009. X3DOM: a DOM-based HTML5/X3D integration model. In Proceedings of the International Conference on $3 D$ Web Technology, Darmstadt, Germany, Jun 16-17 2009 ACM, 127-135. 
Berjon, R., Coloma, D., Froumentin, M., Hanclik, M., Kapyaho, J., Lee, K., Sullivan, B. and Tran, D. 2009. Device APIs Requirements World Wide Web Consortium.

BERKEL, C.V. 1999. Image preparation for 3D LCD. In Proceedings of the SPIE 3639, Stereoscopic Displays and Virtual Reality Systems, San Jose, CA, USA, May 24 1999, 8491.

BERNERS-LEe, T. 1998. Webizing existing systems. In World Wide Web Consortium, personal notes on: Design Issues Architectural and Philosophical Points, Last chage date: March 9, 2010.

CABELlo, R., 2012. three.js / editor [online]. http://threejs.org/editor [Accessed Apr 19 2016].

Chen, Q., WANG, W., WANG, R., Zhang, J. AND LiU, Z. 2014. An approach to support stereoscopic 3D web. In Proceedings of the ACM Symposium on Applied Computing, Gyeongju, Korea, Mar 24-28 2014 ACM, 981-984.

Chistyakov, A., GonzÁlez-ZúÑIGa, D. And CARRabina, J. 2013. Bringing the Web Closer: Stereoscopic 3D Web Conversion. In Proceedings of the Latin American Conference on Human Computer Interaction, Carrillo, Costa Rica, Dec 2-6 2013 Springer International Publishing, 22-25.

DIRKSEN, J. 2013. Learning Three.js: The JavaScript 3D Library for WebGL. Packt Publishing.

Fraser, S., JaCKSON, D., O'ConNOR, E. AND SChUlze, D. 2013. CSS Transforms Module Level 1 World Wide Web Consortium.

GONZÁlEZ-ZÚÑIGA，D. AND CARRABINA，J. 2015. Hacking HTML5 canvas to create a stereo 3D renderer. In Proceedings of the IEEE Symposium on 3D User Interfaces, Arles, France, Mar 23-24 2015 IEEE, 155-156.

HAN, S. AND LEE, D.-Y. 2012. Extensions for Stereoscopic 3D support World Wide Web Consortium.

Hors, A.L., Hégaret, P.L., Wood, L., Nicol, G., Robie, J., Champion, M. And Byrne, S. 2004. Document Object Model (DOM) Level 3 Core Specification World Wide Web Consortium.

Johnston, S., Renambot, L. And SaUter, D. 2013. Employing WebGL to develop interactive stereoscopic 3D content for use in biomedical visualization. In Proceedings of the SPIE 8649, The Engineering Reality of Virtual Reality, Burlingame, CA, USA, Mar 3 2013, 864905.

KIM, D.-W., LeE, K.-H., Yoon, S.K., Kwon, Y.-M., Chang, E.-Y. AND KIM, S.-K. 2012. Analysis and compensation of distorted stereoscopic images due to the observer's viewing orientation. Optics Communications 285, 3909-3916.

Kooima, R.L., Peterka, T., Girado, J.I., Ge, J., Sandin, D.J. And DEFAnT, T.A. 2007. A GPU Sub-pixel Algorithm for Autostereoscopic Virtual Reality. In Proceedings of the IEEE Virtual Reality Conference, Charlotte, NC, USA, Mar10-14 2007 IEEE, 131-137.

LEE, H.-J., YIM, H.-J. AND LIM, S.-B. 2015. Development of Emulator for CSS3 Extension to Represent Web Contents on Stereo Device. International Journal of Smart Home 9, 123134.

PARISI, T. 2012. WebGL: Up and Running. O'Reilly Media.

SeO, D., Yoo, B. AND Ko, H. 2015. Webized 3D experience by HTML5 annotation in 3D web. In Proceedings of the International Conference on $3 \mathrm{D}$ Web Technology, Heraklion, Crete, Greece, Jun 18-21 2015 ACM, 73-80.

Seo, D., Yoo, B. And Ko, H. 2016. Webizing Mixed Reality for Cooperative Augmentation of Life Experience. In Proceedings of the ACM Conference on Computer Supported Cooperative
Work and Social Computing Companion, San Francisco, CA, USA, Feb 27-Mar 32016 ACM, 401-404.

Sons, K., Klein, F., Rubinstein, D., Byelozyorov, S. And SLuSALleK, P. 2010. XML3D: interactive 3D graphics for the web. In Proceedings of the International Conference on Web 3D Technology, Los Angeles, CA, USA, Jul 24-25 2010 ACM, $175-184$

SORBIER, F.D., NOZICK, V. AND BIRI, V. 2008. GPU rendering for autostereoscopic displays. In Proceedings of the International Symposium on 3D Data Processing, Visualization and Transmission, Atlanta, GA, USA, Jun 18-20 2008.

Urey, H., Chellappan, K.V., Erden, E. And Surman, P. 2011. State of the Art in Stereoscopic and Autostereoscopic Displays. Proceedings of the IEEE 99, 540-555.

Vukicevic, V., Jones, B., Gilbert, K. AND WiemeERsch, C.V. 2016. WebVR World Wide Web Consortium.

W3C Community GRoup, 2013. Declarative 3D for the Web Architecture [online]. https://www.w3.org/community/declarative3d [Accessed Apr 7 2016].

W3C Community Group, 2013. Stereoscopic 3D Web [online]. https://www.w3.org/community/stereoweb [Accessed Apr 5 2016].

W3C COMMUNiTy Group, 2016. WebVR [online]. https://www.w3.org/community/webvr [Accessed Apr 7 2016]. 